\title{
1 Epidemiologische und gesundheitsökonomische Aspekte
}

\author{
Thomas Lehnert, Alexander Konnopka, Steffi G. Riedel-Heller \\ und Hans-Helmut König
}

In den letzten drei Jahrzehnten hat sich der Anteil an übergewichtigen Menschen weltweit mehr als verdoppelt. Nach Schätzungen der Weltgesundheitsorganisation (WHO 2011a) sind gegenwärtig mehr als 1,5 Milliarden Menschen übergewichtig, von denen rund ein Drittel - mehr als 200 Millionen Männer und fast 300 Millionen Frauen - als adipös klassifiziert werden muss (s. Box und Tab. 1) (Bessesen 2008; WHO 2011a). In den meisten Ländern der Welt stellen Übergewicht und Adipositas heute eine größere Gesundheitsgefährdung dar als Unterernährung.

\begin{abstract}
Ein diagnostisches Maß für Übergewicht
Das international gebräuchlichste diagnostische Maß für Übergewicht ist der Body-Mass-Index (BMI), der sich aus dem Gewicht geteilt durch die Körpergröße zum Quadrat ergibt $\left(B M I=k g / m^{2}\right)$. Da der BMl einfach zu bestimmen ist und zu 95\% mit der Fettmasse eines Menschen korreliert, stellt er ein brauchbares indirektes Maß der Körperfettmasse dar. Das Ausmaß des Übergewichtes kann mithilfe einer auf dem BMI beruhenden Klassifizierungstabelle der Weltgesundheitsorganisation (WHO 2011b) bestimmt werden (s. Tab. 1). Diese lässt sich jedoch nicht auf Kinder und Jugendliche anwenden, da der BMI hier noch stark von alters- und geschlechtsabhängigen physiologischen Veränderungen der Körpermasse beeinflusst wird. Neben dem BMI existieren weitere, weniger gebräuchliche Messmethoden, die größtenteils auch für Kinder und Jugendliche geeignet sind (Robert-Koch-Institut 2003).
\end{abstract}

Adipositas ist eine chronische Erkrankung, die durch eine starke Erhöhung des Körpergewichts gekennzeichnet ist. Ihr
Tab. 1 Body-Mass-Index

\begin{tabular}{|l|l}
\hline Bezeichnung & BMI $\left(\mathrm{kg} / \mathrm{m}^{2}\right)$ \\
\hline Normalgewicht & $18,5-24,9$ \\
\hline Übergewicht & $25,0-29,9$ \\
\hline Adipositas Grad I (moderat) & $30,0-34,9$ \\
\hline Adipositas Grad II (schwer) & $35,0-39,9$ \\
\hline Adipositas Grad III (extrem) & $\geq 40$ \\
\hline
\end{tabular}

liegt eine über das Normalmaß hinausgehende Vermehrung des Körperfettanteils zugrunde (Bray 1999). Ein stark erhöhter Körperfettanteil hat vielfältige negative Konsequenzen für den menschlichen Organismus und stellt einen Risikofaktor für verschiedene zum Teil schwerwiegende Folgeoder Begleiterkrankungen dar, insbesondere Stoffwechselerkrankungen (z.B. Diabetes mellitus Typ II, Hyperlipidämie, Dyslipidämie), Herz-Kreislauf-Erkrankungen (z.B. Hypertonie, koronare Herzkrankheit, Herzinsuffizienz, Schlaganfall), Erkrankungen des Bewegungsapparats (z.B. Arthrose), und verschiedene Krebserkrankungen (z.B. Brustkrebs, Darmkrebs) (Lenz et al. 2009). Darüber hinaus leiden viele Betroffene unter psychischen Belastungen aufgrund gesellschaftlicher Stigmatisierung und/oder eigener Unzufriedenheit (Robert-Koch-Institut 2003). Mit Übergewicht und Adipositas geht aufgrund der Vielzahl an assoziierten Erkrankungen eine sehr hohe Krankheitslast und erhöhte Mortalität einher. Weltweit sterben jährlich rund 2,8 Millionen Menschen an den Folgen des Übergewichts (WHO 2011a). 


\subsection{Epidemiologische Aspekte}

Obwohl eine weltweite Zunahme von Übergewicht und Adipositas stattfindet, sowohl bei Erwachsenen als auch bei Kindern, sind nicht alle Regionen der Welt gleichermaßen betroffen.

Während die Bevölkerungen verschiedener pazifischer Inseln wie Nauru und Amerikanisch Samoa Adipositasprävalenzen von über $60 \%$ aufweisen, ist in vielen asiatischen Ländern wie beispielsweise Vietnam, Indien und China nur ein sehr kleiner Teil der Bevölkerung (unter 3\%) adipös (WHO 2011b). Unter den entwickelten Ländern sind insbesondere die USA $(33,9 \%)$, Großbritannien $(24,5 \%)$, Kanada $(23,1 \%)$ und Israel $(22,9 \%)$ stark betroffen.

Ein großes Gesundheitsproblem stellt Adipositas auch in wohlhabenden, ölreichen arabischen Staaten dar, z.B. in Saudi-Arabien (35,6\%), den Vereinigten Arabischen Emiraten (33,7\%) und in Kuwait $(28,8 \%)$, in denen sich der Anteil der adipösen Erwachsenenbevölkerung innerhalb der letzten 20 Jahre verdreifacht hat (Bessesen 2008).

Die starke Zunahme der Adipositasprävalenz, insbesondere in Schwellenländern, liegt darin begründet, dass bei der Entstehung von Übergewicht und Adipositas neben biologischen (genetischen) Faktoren insbesondere gesellschaftliche und ökonomische Lebensbedingungen eine bedeutende Rolle spielen (Bray 1999; Robert-Koch-Institut 2003; WHO 2011a; WHO 2011b). So wird die mit gesellschaftlichen Veränderungen einhergehende ständige Verfügbarkeit von energiereichen Nahrungsmitteln gepaart mit einer sedentären (sitzenden) Lebensweise als bedeutende Ursache der weltweiten Zunahme an Übergewicht und Adipositas angesehen. Diese Veränderungen der Lebensgewohnheiten sind in sich wirtschaftlich schnell entwickelnden Ländern mit einer raschen Zunahme an gesellschaftlichem Wohlstand besonders stark bemerkbar. Auch wenn die Ursachen der Entstehung von Adipositas multifaktorieller Natur sind, so ist Übergewicht grundsätzlich die Folge eines Ungleichgewichts zwischen der in den verzehrten Nahrungsmitteln enthaltenen Energiemenge und dem Energieverbrauch einer Person (Bray 1999).

In Europa hat sich der Anteil adipöser Personen in den letzten 20 Jahren annähernd verdoppelt und liegt nun bei $15,5 \%$, mit nur geringen Unterschieden zwischen Männern und Frauen (OECD 2010). Allerdings gibt es auch innerhalb Europas sehr große Unterschiede hinsichtlich des Anteils der betroffenen Bevölkerungsanteile in den einzelnen Ländern (s. Abb. 1). So ist dieser in Großbritannien $(24,5 \%)$ rund dreimal so hoch wie in Rumänien $(7,9 \%)$ oder der Schweiz $(8,1 \%)$ (OECD 2010). In Deutschland sind mehr als die Hälfte aller erwachsenen Personen übergewichtig (36,1\%) oder adipös ( $16 \%$ ) (Robert-Koch-Institut 2011). Unter Kindern und Jugendlichen liegt die Prävalenz von Übergewicht, einschließlich Adipositas je nach Definition zwischen 10\% und 20\% (König u. Konnopka 2008).

Die relativ hohe Prävalenz unter Kindern und Jugendlichen ist besorgniserregend, da Übergewicht und Adipositas bereits im Kindes- und Jugendalter zu psychologischen und somatischen Beeinträchtigungen führen können (Lee 2007) und adipöse Kinder und Jugendliche, im Vergleich zu gleichaltrigen Normalgewichtigen ein deutlich erhöhtes Risiko aufweisen, im Erwachsenenalter noch immer übergewichtig/ adipös zu sein (Wang et al. 2008).

Betrachtet man die Verteilung von Übergewicht und Adipositas nach Altersgruppen, so zeigt sich, dass die Häufigkeit von Übergewicht und Adipositas mit dem Alter stark zunimmt. Außerdem findet sich bei Männern in allen Altersgruppen ein deutlich größerer Anteil an Übergewichtigen, wohingegen Adipositas bei Männern und Frauen in etwa gleich häufig vorkommt (Robert-Koch-Institut 2003).

Auch hinsichtlich des Sozialstatus existieren Unterschiede in der Häufigkeit von Übergewicht und Adipositas. So gehören Männer als auch Frauen mit Übergewicht und Adipositas deutlich häufiger der Unterschicht an, das heißt, sie verfügen über eine geringere Schulbildung, einen niedrigeren beruflichen Status und ein geringeres Einkommen. Besonders stark betroffen sind Frauen ab 30 Jahren mit niedrigem sozio-ökonomischen Status.

\subsection{Gesundheitsökonomische Aspekte}

Mit Übergewicht und Adipositas geht aufgrund der Vielzahl an Folgeerkrankungen eine enorme Krankheitslast einher. Aus Sicht der Gesundheitsökonomie sind neben dem Verlust an Lebensjahren und Lebensqualität insbesondere die durch Übergewicht und Adipositas verursachten Krankheitskosten von Bedeutung. Hierzu gehören zum einen die Kosten der Inanspruchnahme von medizinischen Versorgungsleistungen aufgrund erhöhter Morbidität (direkte Kosten), zum anderen der volkswirtschaftliche Produktionsverlust aufgrund krankheitsbedingter Arbeitsausfallzeiten (indirekte Kosten). 


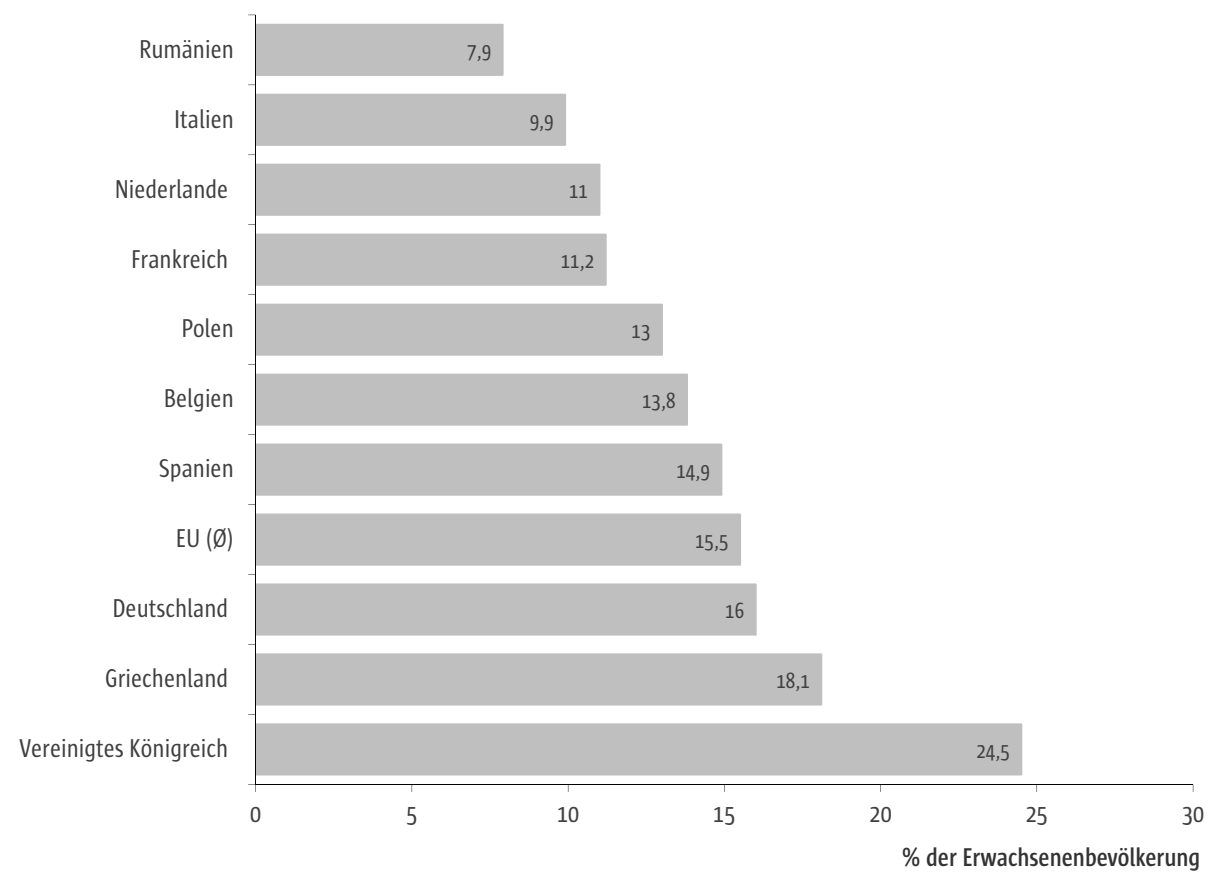

Abb. 1 Anteil adipöser Erwachsener (BMI $\geq 30)$ in den 10 bevölkerungsreichsten Ländern Europas (eigene Darstellung nach Daten aus OECD 2010, Robert-Koch-Institut 2011)

Hinsichtlich der zur Bestimmung der Kosten verwendeten Daten lassen sich zwei Ansätze unterscheiden: Bottom-up-Ansatz und Top-down-Ansatz.

- In Krankheitskostenstudien nach dem Bottom-upAnsatz werden die Kosten für krankheitsbezogene Leistungen bei den betroffenen Patienten gemessen. Der individuelle Ressourcenverbrauch einzelner Patienten wird hierbei durch Befragungen zur Inanspruchnahme von Gesundheitsleistungen oder personenbezogenen Abrechnungsdaten von Versicherten erfasst.

- Erfolgt die Berechnung von Krankheitskosten aus hoch aggregierten Inanspruchnahme- oder Kostendaten (z.B. von statistischen Ämtern oder Finanzierungsträgern), die einzelnen Diagnosegruppen zugeordnet werden, liegt eine Krankheitskostenstudie nach dem sog. Top-Down-Ansatz vor (König u. Konnopka 2008).

\subsection{1 Ökonomische Krankheitslast}

Für die USA wurde in mehreren, nach dem Bottomup-Ansatz durchgeführten Krankheitskostenstudi- en gezeigt, dass adipöse Personen mehr stationäre Krankenhaustage (34\%-74\%), mehr Arztbesuche (14\%-38\%) und mehr Arzneimittelverschreibungen (um den Faktor 1,8 erhöht) als normalgewichtige Personen aufweisen (Quesenberry et al. 1998; Thompson et al. 2001). Aufgrund der höheren Leistungsinanspruchnahme fielen für übergewichtige und adipöse Personen letztlich auch signifikant höhere Pro-Kopf-Gesundheitsausgaben an (Arterburn et al. 2005; Konnopka et al. 2011). Arterburn et al. 2005 berechneten durchschnittliche Pro-Kopf-Gesundheitsausgaben nach BMI-Klassen für die USA im Jahr 200o. Diese lagen für Personen mit einem BMI zwischen 35 und 39, 9 bei 3.511 USD, für Personen mit einem BMI $\geq 40$ sogar bei 4.399 USD, während sie bei Normalgewichtigen lediglich 2.547 USD betrugen. Eine in Deutschland durchgeführte Krankheitskostenstudie (von Lengerke et al. 2006) ermittelte, dass die durchschnittlichen Jahreskosten der medizinischen Versorgung bei Personen mit starker Adipositas (BMI > 35) um circa 200\% höher waren als die Versorgungskosten normalgewichtiger Personen (s. Abb. 2). Für Personen mit Übergewicht(BMI25-29,9) lagen die medizinischen Versorgungskosten sogar 


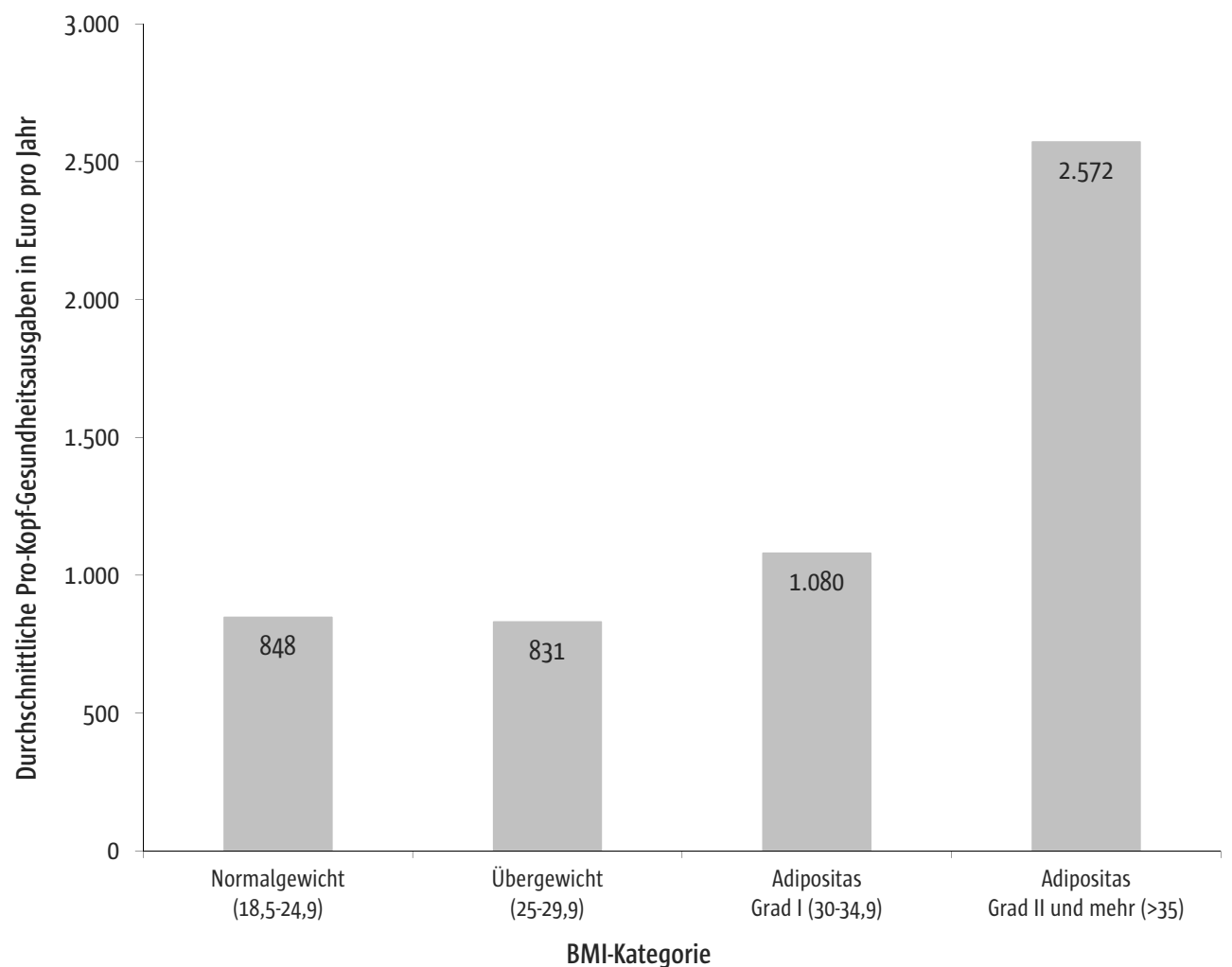

Abb. 2 Pro-Kopf-Gesundheitsausgaben nach BMI-Kategorie in Deutschland im Jahr 2000 (nach von Lengerke et al. 2006)

leicht unterhalb, bei moderater Adipositas (BMI 30-34,9) leicht oberhalb der Kosten von Normalgewichtigen. Die Ergebnisse verschiedener internationaler Krankheitskostenstudien haben gezeigt, dass Adipositas $(\mathrm{BMI} \geq 30)$ im Erwachsenenalter mit durchschnittlich $30 \%$ höheren medizinischen Pro-Kopf-Versorgungskosten assoziiert ist (Withrow u. Alter 2011). Moderate zusätzliche Versorgungskosten fanden sich auch für adipöse Kinder und insbesondere Jugendliche (König et al. 2011).

International liegen zahlreiche nach dem Topdown-Ansatz durchgeführte Studien zu den gesamten durch Übergewicht und Adipositas verursachten Kosten (pro Jahr) vor (König u. Konnopka 2008; Withrow u. Alter 2011). Diese erlauben Aussagen über den Anteil der durch Übergewicht und Adipositas verursachten Versorgungskosten an den gesamten Gesundheitsausgaben eines Landes pro Jahr. Für Deutschland wurden die Gesamtkosten von Adipositas und ihren Folgekrankheiten in zwei Top-down-
Krankheitskostenstudien geschätzt (Sander u. Bergemann 2003; Konnopka et al. 2011). Während Sander und Bergemann (2003) für das Jahr 2001 Cesamtkosten von 4,24 Mrd. EUR berechneten (2,03 Mrd. EUR direkte und 2,21 Mrd. EUR indirekte Kosten), fanden Konnopka und Mitarbeiter (2011) für das Jahr 2002 deutlich höhere adipositas-assoziierte Gesamtkosten von 9,87 Mrd. EUR $(4,85 \mathrm{Mrd}$. EUR direkte und 5,02 Mrd. EUR indirekte Kosten). Der größte Anteil an den Gesamtkosten entfiel dabei auf kardiovaskuläre $(39,9 \%)$ und endokrinologische Erkrankungen (24,8\%) sowie Neubildungen $(25,4 \%$ )(s. Tab. 2). Die Größenordnung des Anteils an den gesamten direkten Gesundheitskosten in Deutschland war im internationalen Vergleich mit 2,1\% noch moderat, wobei der Anteil zwischen $0,7 \%$ in Frankreich und 9,1\% in den USA lag (Königu. Konnopka 2008; Withrow u. Alter 2011; Konnopka et al. 2011). Im Vergleich mit anderen bedeutenden Risikofaktoren kommt Adipositas in Deutschland eine annährend so große Bedeutung wie dem 
Tab. 2 Übergewicht- und Adipositas-attributable Gesamtkosten in Deutschland 2002 ${ }^{\mathrm{a}}$

\begin{tabular}{|c|c|c|c|}
\hline Krankheitsgruppen & $\begin{array}{l}\text { Direkte Kosten in Mio. EUR } \\
\text { (Anteil an gesamten direkten Kosten) }\end{array}$ & $\begin{array}{l}\text { Indirekte Kosten in Mio. EUR } \\
\text { (Anteil an gesamten indirekten Kosten) }\end{array}$ & $\begin{array}{l}\text { Gesamtkosten in Mio. EUR } \\
\text { (Anteil an Gesamtkosten) }\end{array}$ \\
\hline Neubildungen & $660(13,6 \%)$ & $1850(36,9 \%)$ & $2510(25,4 \%)$ \\
\hline $\begin{array}{l}\text { Endokrinologische } \\
\text { Erkrankungen }\end{array}$ & $2077(42,8 \%)$ & $1008(20,1 \%)$ & $3085(31,3 \%)$ \\
\hline $\begin{array}{l}\text { Kardiovaskuläre } \\
\text { Erkrankungen }\end{array}$ & $1836(37,8 \%)$ & $2100(41,9 \%)$ & $3936(39,9 \%)$ \\
\hline $\begin{array}{l}\text { Gastroenterologische } \\
\text { Erkrankungen }\end{array}$ & $282(5,8 \%)$ & $61(1,2 \%)$ & $343(3,5 \%)$ \\
\hline GESAMT & $4854^{b}(100,0 \%)$ & $5019(100,0 \%)$ & $9873(100,0 \%)$ \\
\hline
\end{tabular}

Tabak- und Alkoholkonsum zu, auf welche ein Anteil von $3,3 \%$ und $3,8 \%$ an den gesamten Gesundheitsausgaben zurückgeht (Konnopka et al. 2011).

\subsubsection{Kosteneffektivität präventiver und therapeutischer Maßnahmen}

Aufgrund der mit Übergewicht und Adipositas einhergehenden ökonomischen Krankheitslast haben sowohl präventive als auch therapeutische Gesundheitsleistungen das Potenzial, einen erheblichen Anteil der Morbidität, Mortalität und Krankheitskosten zu vermeiden. Aus gesundheitsökonomischer Sicht ist zu beachten, dass die meisten Maßnahmen (Interventionen) zunächst Kosten verursachen. Erweist sich eine Maßnahme als effektiv, besteht die Hoffnung, dadurch Folgekrankheiten und damit zukünftige Krankheitskosten zu vermeiden. Da die gesellschaftlichen Ressourcen, die für die Gesundheitsversorgung zur Verfügung stehen, begrenzt sind, stellt sich für neue Gesundheitstechnologien stets die Frage nach ihrer Kosteneffektivität. Die Beurteilung der Kosteneffektivität ist die Voraussetzung für eine effiziente Verwendung von knappen Ressourcen und kann die Prioritätensetzung in der Gesundheitsversorgung unterstützen.

Prinzipiell lässt sich bei Übergewicht und Adipositas bezüglich des Inhalts von Gesundheitsleistungen zwischen präventiven und therapeutischen Interventionen unterscheiden. Während sich präventive Interventionen an die Allgemeinbevölkerung oder spezielle Risikogruppen richten, zielen therapeutische Interventionen auf eine Reduktion des
Körpergewichtes und/oder der mit Adipositas assoziierten Morbidität bei bereits übergewichtigen oder adipösen Patienten ab. Das Ziel der meisten präventiven als auch therapeutischen Interventionen besteht in einer Modifikation des Ernährungs- und/ oder Bewegungsverhaltens, entweder durch Aufklärung und Schulung, oder indem Risikofaktoren direkt, im Rahmen regulatorischer Maßnahmen, beeinflusst werden (zum Beispiel durch Werbeverbote oder die gezielte Besteuerung ungesunder Lebensmittel). Therapeutische Interventionen können darüber hinaus aus pharmakologischen oder chirurgischen Eingriffen bestehen, die jedoch nur für bestimmte Gruppen (schwer) adipöser Personen infrage kommen.

Die gesundheitsökonomische Evidenz zu präventiven und therapeutischen Interventionen ist eher fragmentarisch (Bachman 2007; Klarenbach et al. 2010; Loveman et al. 2011; Saha et al. 2010). Probleme hinsichtlich der Synthese der Ergebnisse bereiten zum einen die Abgrenzung von Interventionen zur Prävention anderer Krankheiten, da typische Risikofaktoren der Adipositas, wie Ernährungs- und Bewegungsverhalten, auch Risikofaktoren für eine Reihe weiterer Krankheiten darstellen. Zum anderen besteht eine methodische Heterogenität der ökonomischen Evaluationsstudien, weshalb insbesondere die Wahl der Vergleichsalternative (Komparator) problembehaftet ist.

Für die Behandlung stark adipöser Patienten haben sich allerdings verschiedene Varianten bariatrisch-chirugischer Eingriffe, z.B. laparoskopisches Magenband, Roux-En-Y Magenbypass, als die wirksamsten und wirtschaftlichsten Therapien erwiesen (Bult et al. 
2008; Klarenbach et al. 2010). Aufgrund des starken und lang anhaltenden Cewichtsverlusts gehen bariatrische Verfahren, im Vergleich mit der üblichen Behandlung („usual care“) dieser Patientengruppe, trotz relativ hoher Kosten für die Operation selbst (Interventionskosten), langfristig betrachtet mit einer günstigen Kosteneffektivität einher (Klarenbach et al. 2010). Der Grund liegt vor allem in der deutlich verbesserten Lebensqualität der behandelten Patienten, welche aufgrund einer, durch den Gewichtsverlust bedingten, verbesserten Morbiditätsund Gesundheitssituation zustande kommt. Dadurch weisen diese Patienten langfristig betrachtet geringere Versorgungskosten auf, welche das Potenzial besitzen, die hohen Interventionskosten zu kompensieren. Da diese operativen Eingriffe zu schweren Komplikationen führen können, muss eine sorgfältige Auswahl der infrage kommenden stark adipösen Patienten (BMI $\geq 40$ oder BMI $\geq 35$ mit schwerwiegenden Komorbiditäten) vorgenommen werden (Bult et al. 2008).

\section{Zusammenfassung}

Weltweit lässt sich seit einigen Jahrzehnten in allen Altersgruppen eine deutliche Zunahme von Übergewicht und Adipositas beobachten (WHO 2011a). In Deutschland sind heute bereits mehr als die Hälfte aller erwachsenen Personen übergewichtig oder adipös. Die Häufigkeit nimmt mit dem Alter stark zu. Unabhängig vom Alter sind Menschen mit geringerem sozio-ökonomischen Status (geringer Bildung und niedrigem Einkommen) besonders stark betroffen (Robert-Koch-Institut 2003). Diese Entwicklungen sind besorgniserregend, weil Übergewicht und insbesondere Adipositas Risikofaktoren für eine Vielzahl von Begleit- und Folgeerkrankungen darstellen. Neben psychischen Belastungen begünstigen sie bei den Betroffenen vor allem die Entstehung von Krankheiten des Kreislaufsystems, Typ-2-Diabetes, Arthrose und Neubildungen (Lenz et al. 2009). Aufgrund der hohen Morbidität und Mortalität verursachen Übergewicht und Adipositas eine erhebliche medizinische und ökonomische Krankheitslast (Quesenberry et al. 1998).

Die Ergebnisse gesundheitsökonomischer Studien zeigen, dass mit Adipositas erhebliche Kosten für das Gesundheitssystem und die Gesellschaft verbunden sind, wobei nur ein kleiner Teil der medizinischen (direkten) Versorgungskosten aufgrund des Übergewichts/Adipositas selbst zustande kommen. Der Großteil dieser Kosten lässt sich der Behandlung von Folgeerkrankungen zuschreiben. Rund die Hälfte der gesamten Krankheitskosten der Adipositas machen die indirekten Kosten aus. Indirekte Kosten quantifizieren den durch den Verlust an Lebensjahren und Lebensqua- lität zustande kommenden volkswirtschaftlichen Produktionsausfall. Die Evidenz zur Wirtschaftlichkeit von Maßnahmen, welche eine Reduktion der mit Übergewicht und Adipositas verbundenen Krankheitslast anstreben, ist eher fragmentarisch. Obwohl in der internationalen Literatur eine Vielzahl an ökonomischen Evaluationen zu diesem Thema existiert (Bachman 2007; Klarenbach et al. 2010; Loveman et al. 2011; Saha et al. 2010), fällt die Synthese der Ergebnisse aufgrund von methodischen Unterschieden der entsprechenden Studien schwer. Grundsätzlich ist die Wirtschaftlichkeit von präventiven als auch therapeutischen Interventionen bei Übergewicht und Adipositas insbesondere von den Kosten der Intervention selbst, den durch die Intervention bewirkten Gewichtsverlust (und dessen Dauerhaftigkeit), sowie den damit verbundenen Effekten auf Morbidität (Vermeidung von Folgekrankheiten), Lebensqualität, Mortalität und den daraus resultierenden medizinischen Versorgungskosten abhängig.

\section{Literatur}

Arterburn DE, Maciejewski ML, Tsevat I (2005) Impact of morbid obesity on medical expenditures in adults. Int I Obes 29, 334339

Bachman KH (2007) Obesity, weight management, and health care costs: a primer. Dis Manag 10, 129-137

Bessesen DH (2008) Update on obesity. I Clin Endocrinol Metab 93, 2027-2034

Bray GA (1999) Etiology and pathogenesis of obesity. Clin Cornerstone 2, 1-15

Bult M), van Dalen T, Muller AF (2008) Surgical treatment of obesity. Eur Endocrinol 158, 135-145

Klarenbach S, Padwal R, Wiebe N, Hatzel M, Birch D, Manns B, Karmali S, Sharma A, Tonelli M (2010) Bariatric surgery for severe obesity: systematic review and economic evaluation: Canadian Agency for Drugs and Technologies in Health. Technology report; no. 129 http://www.cadth.ca/media/pdf/H0485_Bariatric_Surgery_ for_Severe_Obesity_tr_e.pdf (abgerufen am 11. April 2011)

König HH, Konnopka A (2008) Gesundheitsökonomische Aspekte von Übergewicht und Adipositas. Adipositas 2, 198-203

König HH, Lehnert T, Riedel-Heller S, Konnopka A (2011) Prävention und Therapie von Übergewicht und Adipositas im Kindes- und Jugendalter aus gesundheitsökonomischer Sicht. Bundesgesundheitsbl Gesundheitsforsch Gesundheitsschutz 54, 611-620

Konnopka A, Bodemann M, König HH (2011) Health burden and costs of obesity and overweight in Germany. Eur J Health Econ $12,345-352$

Lee WW (2007) An overview of pediatric obesity. Pediatr Diabetes 8 Suppl 9, 76-87

von Lengerke T, Reitmeir P, John I (2006) Direkte medizinische Kosten der (starken) Adipositas: ein Bottom-up-Vergleich über-vs. normalgewichtiger Erwachsener in der KORA-Studienregion. Gesundheitswesen 68, 110-115 
Lenz M, Richter T, Mühlhauser I (2009) The morbidity and mortality associated with overweight and obesity in adulthood: a systematic review. Dtsch Arztebl Int 106, 641-648

Loveman E, Frampton GK, Shepherd I, Picot I, Cooper K, Bryant I, Welch W, Clegg A (2011) The clinical effectiveness and cost-effectiveness of long-term weight management schemes for adults: a systematic review. Health Technol Assess 15, 1-182

OECD (2010) Health at a glance: Europe 2010. URL: http://dx.doi. org/10.1787/health_glance-2010-en (abgerufen am 11. April 2011)

Quesenberry CP, Caan B, Jacobson A (1998) Obesity, health services use, and health care costs among members of a health maintenance organization. Arch Intern Med 158, 466-472

Robert-Koch-Institut (2003) Übergewicht und Adipositas. Gesundheitsberichterstattung des Bundes Heft 16. Berlin: RKI

Robert-Koch-Institut (2011) Daten und Fakten: Ergebnisse der Studie „Gesundheit in Deutschland aktuell 2009“, Gesundheitsberichterstattung des Bundes. Berlin: RKI

Saha S, Gerdtham UG, Johansson P (2010) Economic evaluation of lifestyle interventions for preventing diabetes and cardiovascular diseases. Int | Environ Res Public Health 7, 3150-3195
Sander B, Bergemann R (2003) Economic burden of obesity and its complications in Germany. Eur J Health Econ 4, 248-253

Thompson D, Brown JB, Nichols GA, Elmer PI, Oster G (2001) Body mass index and future healthcare costs: A retrospective cohort study. Obes Res 9, 210-218

Wang LY, Chyen D, Lee S, Lowry R (2008). The association between body mass index in adolescence and obesity in adulthood. I Adolesc Health 42, 512-518

WHO (2011a) Obesity and overweight URL: http://www.who.int/ mediacentre/factsheets/fs311/en/index.html (abgerufen am 11. April 2011)

WHO (2011b) Global database on body mass index (BMI). URL: http:// apps.who.int/bmi/index.jsp (abgerufen am 11. April 2011)

Withrow D, Alter DA (2011) The economic burden of obesity worldwide: a systematic review of the direct costs of obesity. Obes Rev 12, 131-41

\section{Danksagung}

Diese Arbeit wurde durch das Bundesministerium für Bildung und Forschung (BMBF), FKZ: 01E01001, gefördert.

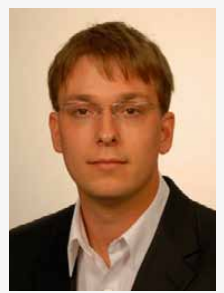

\section{Dipl.-Soz. Thomas Lehnert}

Nach dem Studium der Soziologie und Rechtswissenschaft als Mitarbeiter am Lehrstuhl für Gesundheitsökonomie (Universität Leipzig) tätig. Anschließend Wechsel ans Institut für Medizinische Soziologie, Sozialmedizin und Gesundheitsökonomie am Universitätsklinikum Hamburg-Eppendorf (UKE). Seit 2011 Projektmitarbeiter des IFB AdipositasErkrankungen (Leipzig) sowie Mitglied des Hamburg Center of Health Economics (HCHE).

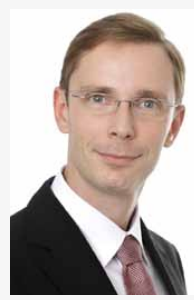

\section{Dr. Alexander Konnopka}

Dr. Alexander Konnopka ist Wissenschaftler im Forschungsbereich Gesundheitsökonomie des Instituts für Medizinische Soziologie, Sozialmedizin und Gesundheitsökonomie (IMSG) am Universitätsklinikum Hamburg-Eppendorf (UKE). Er studierte Medizin an der Universität Leipzig und Volkswirtschaftslehre an der FernUniversität Hagen. Dr. Konnopka war von 2006 bis 2010 wissenschaftlicher Mitarbeiter am Lehrstuhl für Gesundheitsökonomie des Universitätsklinikums Leipzig und wechselte 2010 an das Universitätsklinikum HamburgEppendorf. Neben seiner Tätigkeit als Arbeitsgruppenleiter in Forschung und Lehre an der Medizinischen Fakultät ist Dr. Konnopka auch aktiv am Aufbau des Hamburg Center for Health Economics beteiligt.

Die Forschungsschwerpunkte von Dr. Konnopka liegen im Bereich der empirischen und der modellgestützten Messung und Analyse von Krankheitskosten und der Kosten-Effektivität von Gesundheitsleistungen. 


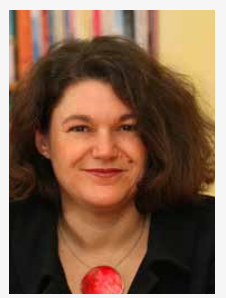

\section{Prof. Dr. med. Steffi G. Riedel-Heller, MPH}

Ausbildung zur Psychiatriepflegerin, danach Medizinstudium in Leipzig. Ärztin in Weiterbildung an der Klinik und Poliklinik für Psychiatrie der Universität Leipzig, Zusatzausbildung zur Gesundheitswissenschaftlerin (Master of Public Health) an der Johns Hopkins University in Baltimore, USA. Klinische Tätigkeit, Forschung und Lehre an der Klinik und Poliklinik für Psychiatrie der Universität Leipzig, 2002 Facharztanerkennung und Habilitation. 2004 bis 2010 C3-Professorin für Public Health und seit 2010 W3-Professorin und Direktorin des Instituts für Sozialmedizin, Arbeitsmedizin und Public Health an der Medizinischen Fakultät der Universität Leipzig. Forschungsschwerpunkte: Epidemiologie psychischer und somatischer Erkrankungen im Alter, Prävention und Versorgungsforschung.

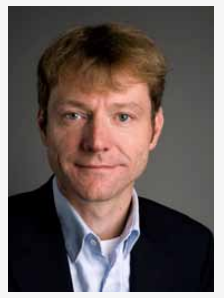

\section{Prof. Dr. Hans-Helmut König}

Prof. Dr. Hans-Helmut König ist Direktor des Instituts für Medizinische Soziologie, Sozialmedizin und Gesundheitsökonomie und Inhaber des Lehrstuhls für Gesundheitswissenschaftliche Versorgungsforschung und Gesundheitsökonomie am Universitätsklinikum Hamburg-Eppendorf/Medizinische Fakultät der Universität Hamburg. Seine Forschungsschwerpunkte liegen in den Bereichen Gesundheitsökonomie, Versorgungsforschung und Gesundheitssystemforschung. 